\title{
Sacroiliitis - early diagnosis is key
}

This article was published in the following Dove Press journal:

Journal of Inflammation Research

\section{Gleb Slobodin 1,2 \\ Haya Hussein' \\ Itzhak Rosner ${ }^{1,2}$ \\ Iris Eshed ${ }^{3,4}$}

'Rheumatology Unit, Bnai Zion Medical Center, Haifa, Israel; ${ }^{2}$ Ruth and Bruce Rappaport Faculty of Medicine, Technion, Haifa, Israel; ${ }^{3}$ Department of Diagnostic Imaging, Sheba Medical Center, Tel Aviv, Israel; ${ }^{4}$ Sackler Faculty of Medicine, Tel Aviv University, Tel Aviv, Israel
Correspondence: Gleb Slobodin Rheumatology Unit, Bnai Zion Medical Center, 47 Golomb St, Haifa, 3804I, Israel

Email gslobodin@yahoo.com

\begin{abstract}
Sacroiliitis, inflammation of the sacroiliac joint (SIJ), may be associated with many rheumatic and nonrheumatic disorders. The diagnosis of sacroiliitis may be difficult in many patients, and awareness of its typical manifestations along with recognition of its diverse presentations and cognizance of the limitations of today's imaging modalities are critical to good clinical practice. This review presents the didactic approach to the early diagnosis of sacroiliitis in patients with suspected axial spondyloarthritis and other nosologic entities, discussing also differential interpretation of information acquired from patients' histories, physical examination, and imaging. Keywords: sacroiliitis, axial spondyloarthritis, diagnosis, imaging, Sacroiliac joint, differential diagnosis
\end{abstract}

\section{Introduction}

Sacroiliitis, inflammation of the sacroiliac joint (SIJ), is a primary manifestation of axial spondyloarthritis (AxSpA) and may be seen in many other rheumatic and nonrheumatic disorders as well (Table 1). As the symptoms of sacroiliac involvement at presentation are not specific, the diagnosis of sacroiliitis is heavily dependent on confirmatory imaging. The various imaging modalities, however, also have their limitations in both sensitivity and specificity, leading to inconclusive descriptions and reports in some patients. Thus, to arrive at good medical practice, one needs knowledge of the characteristic clinical manifestations of sacroiliitis together with recognition of its diversity in presentation and awareness of the limitations of the presently available imaging tools. This review provides a didactic approach to the early diagnosis of sacroiliitis in patients with suspected $\mathrm{AxSpA}$, discussing differential interpretation of data acquired from patients' histories, physical examination, and imaging.

\section{Sacroiliac pain}

Sacroiliac pain is the hallmark of SIJ involvement in the inflammatory process. Classical inflammatory back pain (IBP) of AxSpA is characterized by gradual onset and daily appearance of low back or buttock pain in young individuals, with typical prominence at night and associated stiffness upon awakening, usually with some amelioration after exercise. Based on these features, a variety of criteria for the differentiation of IBP/ sacroiliac pain from the much more prevalent "mechanical back pain" have been suggested. ${ }^{1}$ However, criteria developed for purposes of classification, intended initially for inclusion of subjects into studies, may not apply in daily practice. As such, in a study 
Table I Medical conditions, involving SIJ

\begin{tabular}{|c|c|c|c|}
\hline Rheumatic inflammatory diseases & Rheumatic noninflammatory diseases & Infectious diseases & Other diseases \\
\hline Ankylosing spondylitis and other SpA & Osteitis condensans ilii & Pyogenic sacroiliitis & Hyperparathyroidism \\
\hline Familial Mediterranean fever & Diffuse idiopathic skeletal hyperostosis & Brucellosis & Lymphoma and other malignancies \\
\hline Bechet's disease & Osteoarthritis & Tuberculosis & \\
\hline $\begin{array}{l}\text { SAPHO (synovitis, acne, pustulosis, } \\
\text { hyperostosis, osteitis) }\end{array}$ & Multicentric reticulohistiocytosis & Whipple disease & \\
\hline Gout and pseudogout & & & \\
\hline
\end{tabular}

Abbreviations: SIJ, sacroiliac joint; SpA, spondyloarthritis.

of 322 patients with back pain, 113 of whom had AxSpA, the sensitivity of the predefined set of criteria composed of morning stiffness of $>30 \mathrm{~min}$, improvement by movement/not by rest, waking up in the second half of the night because of back pain, and improvement with nonsteroidal anti-inflammatory drugs was only $52.2 \% .^{2}$ Hence, exclusion of the diagnosis of AxSpA just based on failure to satisfy one or another set of IBP classification criteria may misdiagnose a large proportion of patients. Instead, the presence of even 1 or 2 IBP features, particularly if unexplained by an alternative diagnosis and especially when accompanied by other SpA-related features, such as enthesitis or psoriasis, should trigger further work up for $\mathrm{SpA}$. Furthermore, limitation on the age of pain onset, set at 30 years by Berlin criteria and 40 years by the Calin and Assessment of Spondyloarthritis International Society (ASAS) criteria for IBP, should be applied cautiously in clinical practice, particularly in light of recent reports of late-onset $\mathrm{SpA} .^{3}$

Of importance, it should be remembered that many patients with AxSpA, particularly at early stages of the disease, can present with atypical acute, intermittent or unilateral pain originating from the SIJ, which may be misinterpreted as secondary to a mechanical cause. In addition, while typical location of the sacroiliac pain is usually limited to buttock and lower lumbar regions, radiation to the groin, lower abdomen, trochanter, and even lower leg are reported in some patients. It is believed that diverse innervation of the SI region and involvement of adjacent structures, such as surrounding ligaments, with location of the disease process in different sites within the SIJ may be the reason for this variability in SIJ pain. ${ }^{4}$

Physical examination of the SIJ includes joint palpation and provocation tests aimed to induce pain while stressing the SIJ. The 3 most frequently used provocation tests include the pelvis rock test, FABERE (Flexion, ABduction, External Rotation, Extension), and Gaenslen maneuvers. During the pelvic rock test, a physician places his hands over patient's iliac crests with thumbs held on the anterior superior iliac spines and then compresses the pelvis toward the midline.
The FABERE test can provoke hip pain during the first 3 movements, but after the hip joint is stabilized by FABER, the Extention part, caused by the pressure over the flexed knee joint and the opposite anterior superior iliac spine, stresses the SIJ and can exacerbate SIJ pain. During the Gaenslen maneuver, a patient lies supine on the edge of the couch with both legs bent to his chest and then drops 1 extended leg down the side of the couch to a hip hyperextension position, exerting stress on the SIJ. These provocation tests can be sensitive enough to detect SIJ pain and can further confirm the diagnosis of SIJ disease but have low specificity and may lead to false-positive conclusions if unsupported by further investigations.

\section{Conventional radiography of sacroiliitis}

Traditionally, imaging of the SIJ starts with a roentgenogram (X-ray). However, changes of the SIJ seen on X-ray films are not sensitive or specific enough, at least in the early stages of sacroiliitis. It may take years of clinically evident disease until clear SIJ abnormalities become visible on conventional radiography in many patients with AxSpA..$^{5}$ In these patients, the typical dynamics of changes start with ill-defined sclerosis adjacent to the joint line, which may become dense and extensive, particularly on the iliac side; irregularities of joint end-plates with erosions and widening of SIJ space follow and eventually convert to joint space narrowing with bridging and subsequent ankylosis of the joint. Nevertheless, the interpretation of X-ray films of the SIJ can be difficult and equivocal in SpA, particularly in its early stages. Even radiologists and rheumatologists experienced in the reading of X-ray films of SIJ demonstrate at best moderate agreement on the recognition of radiographic sacroiliitis. ${ }^{6}$ It should be remembered that only a minority of AxSpA patients presenting with new low back or sacroiliac pain will already have clear-cut radiographic sacroiliitis. On the other hand, confounding pseudoankylosis of the SIJ due to anterior bony bridges can be seen on posteroanterior radiographs in patients 
with diffuse idiopathic skeletal hyperostosis. Iliac and sometimes sacral triangular shaped sclerosis about the SIJ, which is the hallmark of osteitis condensans ilii can mimic the sclerosis of inflammatory sacroiliitis. Bone resorption resulting from hyperparathyroidism (primary or secondary) also needs to be differentiated from erosive changes related to SpA. Finally, unilateral sclerosis or erosions adjacent to SIJ in a patient with febrile disease and new back pain may be the presentation of subacute septic sacroiliitis. ${ }^{7}$

\section{Radionuclide methods in the diagnosis of sacroiliitis}

In a review published in 2008, the sensitivity of bone scintigraphy for detection of sacroiliitis in patients with AS was estimated at about $50 \%$ at best. $^{8}$ The true negative scintigraphy in patients with back pain due to mechanical causes (specificity) was calculated as $78 \%$, resulting in relatively low likelihood ratio of between 2.5 and 3 when scintigraphy was used for this diagnostic purpose, significantly lower than that of magnetic resonance imaging (MRI), estimated as up to 10.4 , depending on the MRI feature assessed. ${ }^{9}$ Of relevance, compared to the earlier, more inflammatory stages of disease, the yield of bone scintigraphy for the diagnosis of sacroiliitis is even lower at the ankylosis stage when SIJs are fixed or practically absent. On the other hand, concomitant use of single-photon emission computed tomography, able to confine the uptake area to the SIJ, can significantly improve the diagnostic performance of bone scintigraphy for sacroiliitis. ${ }^{10}$ In counterpoint, the use of radionuclide tools for the diagnosis of sacroiliitis with its radiation exposure for the diagnosis in young patients may be unjustified or even unethical, when MRI, a modality with no radiation exposure and higher sensitivity and specificity is available, and thus its use is not advisable in daily clinical practice. In general, it seems that patients with suspected acute, particularly infectious sacroiliitis can benefit most from the diagnostic abilities of bone scintigraphy for disease localization, while patients with a more indolent course should probably be referred to alternative means of imaging. It also should be remembered that the other noninflammatory conditions involving SIJ, such as osteitis condensans ilii or degenerative disease of SIJ can sometimes manifest with low-grade tracer uptake on bone scintigraphy, necessitating differential approach to the positive examination.

\section{Computed tomography}

Computed tomography (CT) is an excellent imaging modality offering great 3-dimensional, multiplanar, high-resolution images, and thus offers more sensitive, accurate and detailed information compared to plain radiography. ${ }^{11,12}$ However, since like bone scintigraphy, radiation exposure of CT is much higher compared to plain radiography, it is currently not advisable to use pelvic or spinal $\mathrm{CT}$ for diagnosis or follow-up evaluation of the structural SpA-related changes in the SIJs and spine. ${ }^{13}$

Still, many SpA patients undergo lumbar spine CT during their orthopedic low back pain workup process and, if present, valuable data can be obtained from that CT as the SIJs tend to be encompassed on routine CT examinations of the lumbar spine. ${ }^{14}$ Structural lesions seen on CT develop at the later stage of sacroiliitis and include erosions, sclerosis, and ankylosis, of which erosions are the most sensitive and specific for the diagnosis of sacroiliitis (Figure 1). ${ }^{15}$ The CT superiority in the detection of structural findings is especially important in the face of new emerging techniques such as low-radiation CT and dual-energy CT that may lead to a surge in the use of CT. ${ }^{16,17}$ Indeed, in comparison with low-dose CT as the gold standard, conventional radiography missed more than half of the patients and two-thirds of the joints with structural changes consistent with (radiographic) AxSpA on low-dose $\mathrm{CT} .{ }^{16}$ In addition, low-dose, dual-energy CT has a promising role in detecting bone marrow edema (BME) of the spine and the SIJs. ${ }^{17}$ Currently, BME can only be detected by MRI, and the detection of this early sign of inflammation of AxSpA using a fast, readily available technique that does not jeopardize the health of young patients with its use of low radiation exposure, may completely change the imaging workup of patients with SpA.

Tomosynthesis, a method performing high-resolution limited-angle tomography at radiation dose levels about twice that of conventional radiography and almost one tenth that of a CT scan, has recently been reported as able to visualize morphologic changes in the SIJs not seen on plain radiographs. ${ }^{18}$

\section{MRI}

MRI of the SIJs and of the spine has revolutionized diagnosis of early SpA.

With its high contrast resolution, it is able to detect inflammation of the joints in its early stage before structural damage occurs (Figure 1). With potentially effective treatment to be used in a narrow window of opportunity for disease control, MRI was rapidly embraced as a dominant diagnostic tool and at the same time included into the classification criteria for $\mathrm{AxSpA}$, becoming the cornerstone of SpA diagnosis. However, the use of BME as a single diagnostic tool, though easy to apply, has several inherent disadvantages. Mainly, 

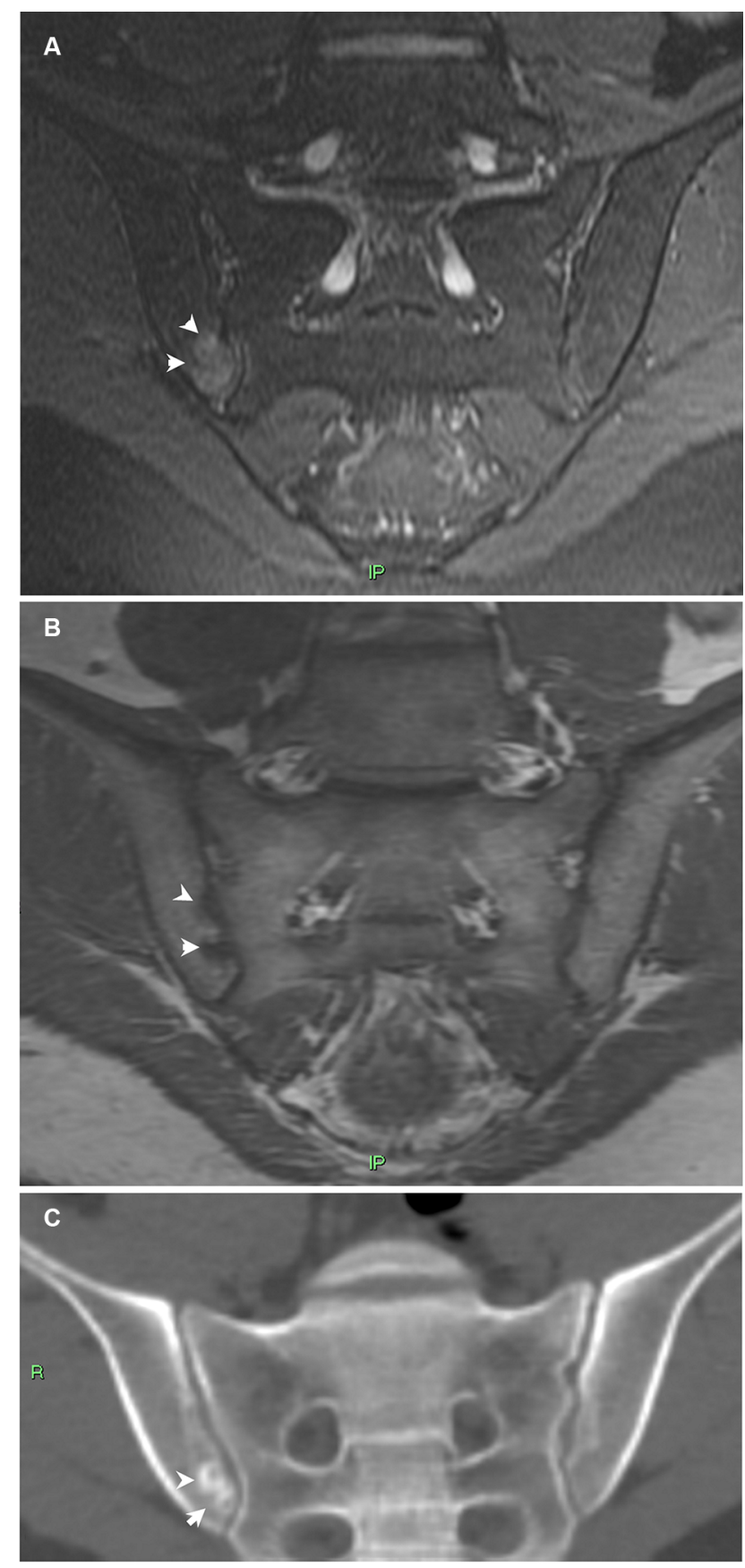

Figure I Early sacroiliitis.

Notes: Semicoronal MRI T2-weighted with fat saturation (A), TI-weighted (B) and semicoronal CT reconstruction (C) images of the SIJs of a 21 year old male with early sacroiliitis. BME is clearly seen on the right iliac side of the joint (arrowheads in A) as well as small erosions at the same location (arrowheads in $\mathbf{B}$ and $\mathbf{C}$ ). Abbreviations: BME, bone marrow edema; CT, computed tomography; MRI, magnetic resonance imaging; SIJ, sacroiliac joint.

minor BME may be seen in many clinical situations and even in healthy individuals, thus a certain threshold of BME and pattern recognition (ie, differentiating between specific disease entities based on the pattern of BME distribution along the joint) needs to be employed when diagnosing inflammatory sacroiliitis on MRI. ${ }^{19}$ Therefore, the potential for false-positive MRI diagnosis of sacroiliitis is relatively high, requiring experience and in-depth radiological experience of the MRI pattern of sacroiliitis and its differential diagnosis. The presence of characteristic structural lesions on MRI improves diagnostic accuracy and was therefore advocated as a consideration in the ASAS classification criteria update. ${ }^{20,21}$ No objective gold standard is available for the diagnosis of AxSpA and thus diagnostic accuracy of MRI for the detection of inflammatory disease is almost impossible to measure. On the one hand, diagnosis is based on expert opinion, and on the other hand, experts' opinions rely heavily on MRI results. The result is a relative low sensitivity and specificity of MRI for the diagnosis of SpA..$^{20,22}$ Thus, this means that while MRI has an important role in the diagnosis of SpA, it cannot and should not be used as a solitary diagnostic tool and the data it supplies should be considered and integrated into the complex of all of a patient's clinical data.

Whole-spine (Figure 2) and whole-body MRI techniques have been added recently as radiological tools enabling screening of the entire spine and SIJ or the entire body for inflammatory involvement of joints and entheses. It is currently mainly used in research, while its clinical benefit for diagnostic and follow-up purposes needs to be evaluated. ${ }^{23}$

\section{Imaging-negative sacroiliitis}

A clinician always feels more comfortable when the diagnosis, particularly the diagnosis of a condition where no unequivocal physical examination or laboratory test exists, will be confirmed by imaging. Sacroiliitis is a good example. Physical examination of a patient with sacroiliitis is never $100 \%$ diagnostic, as every manifestation of sacroiliitis can be mimicked by other pelvic or low back diseases. Conventional radiography is frequently normal in early sacroiliitis. Similarly, bone scintigraphy has low sensitivity, and MRI can miss up to one-third of the patients with AxSpA as well. ${ }^{9,24}$ With this data in mind, the exclusion of the diagnosis of sacroiliitis merely based on negative imaging would mean failure to diagnose about $30 \%$ of patients with early $\mathrm{AxSpA}$ in a timely manner, along with an unknown percentage of patients with alternative diagnoses manifesting with sacroiliitis. Thus, the report of negative imaging should not replace clinical considerations and a reasoned approach to the diagnosis. The example of such an organized approach to the diagnosis of AxSpA, a disease where sacroiliitis is a cornerstone of the diagnosis, was suggested $>10$ years ago. ${ }^{25}$ This approach is based on the presence of characteristic clinical and laboratory features of the disease allowing for 


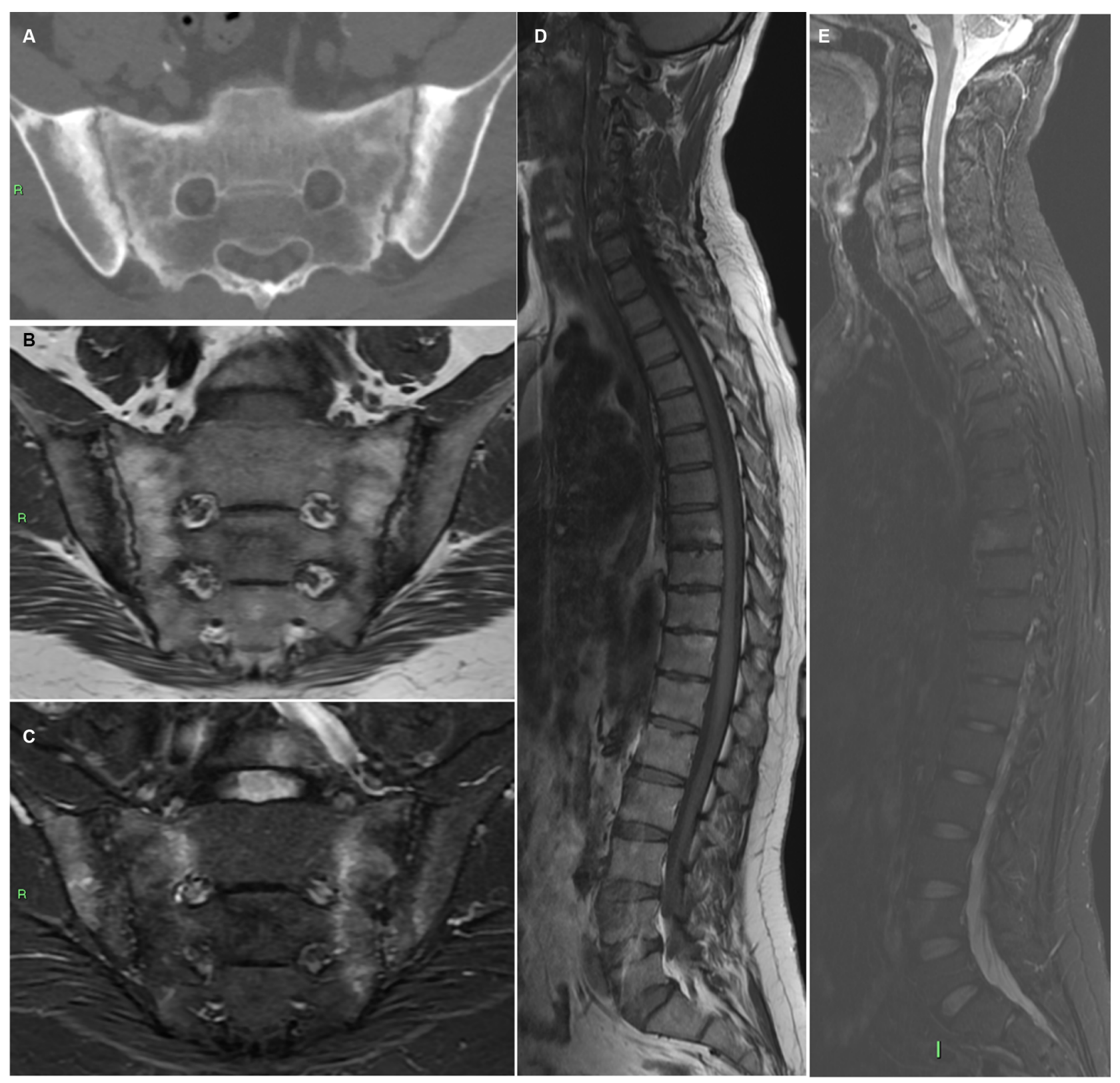

Figure 2 Forty two year old male with ankylosing spondylitis.

Notes: Axial CT image of the SIJs (A) demonstrating advanced disease with bilateral subchondral sclerosis, erosions, and pseudo-widening of the joints. Whole-spine MRI of the same patient with semicoronal TI-weighted (B), STIR (C) of the SIJs, and sagittal TI-weighted (D) and STIR (E) of the entire spine demonstrating bilateral SIJ's BME, fat metaplasia, and erosions as well as corner inflammatory lesions and fatty lesions in the spine.

Abbreviations: BME, bone marrow edema; CT, computed tomography; MRI, magnetic resonance imaging; SIJ, sacroiliac joint; STIR, Short-TI inversion recovery.

diagnosis in the absence of any positive imaging of sacroiliitis in patients with enough indirect evidence of the disease. ${ }^{25}$ Accordingly, the 2009 ASAS classification criteria allow classification of patients with AxSpA without any imaging evidence of sacroiliitis. ${ }^{24}$ Of course, alternative diagnoses should always be taken into consideration during the diagnostic process, particularly in patients without direct disease evidence. In this regard, patients with clinical suspicion of sacroiliitis, but without confirmatory imaging or alternative diagnosis, invariably remain a challenge for a rheumatologist and necessitate repeated evaluations, which may sometimes eventually deliver the desired "proof." A positive response to administration of a specific treatment agent, such as biologic agents usually effective in patients with AxSpA, can serve an additional piece of evidence for existence of the disease. ${ }^{26}$

\section{Disclosure}

The authors report no conflicts of interest in this work. 


\section{References}

1. Weisman MH. Inflammatory back pain. Rheum Dis Clin North Am. 2012;38(3):501-512.

2. Braun A, Saracbasi E, Grifka J, Schnitker J, Braun J. Identifying patients with axial spondyloarthritis in primary care: how useful are items indicative of inflammatory back pain? Ann Rheum Dis. 2011;70(10):1782-1787.

3. Olivieri I, D'Angelo S, Padula A, Leccese P, Palazzi C. Spondyloarthritis with onset after age 45. Curr Rheumatol Rep. 2013;15(12):374.

4. Vleeming A, Schuenke MD, Masi AT, Carreiro JE, Danneels L, Willard FH. The sacroiliac joint: an overview of its anatomy, function and potential clinical implications. J Anat. 2012;221(6):537-67.

5. Said-Nahal R, Miceli-Richard C, Berthelot JM, et al. The familial form of spondylarthropathy: a clinical study of 115 multiplex families. Groupe Français d'Etude Génétique des Spondylarthropathies. Arthritis Rheum. 2000;43(6):1356-1365.

6. van den Berg R, Lenczner G, Feydy A, et al. Agreement between clinical practice and trained central reading in reading of sacroiliac joints on plain pelvic radiographs. Results from the DESIR cohort. Arthritis Rheumatol. 2014;66(9):2403-2411.

7. Slobodin G, Lidar M, Eshed I. Clinical and imaging mimickers of axial spondyloarthritis. Semin Arthritis Rheum. 2017;47(3):361-368.

8. Song IH, Carrasco-Fernández J, Rudwaleit M, Sieper J. The diagnostic value of scintigraphy in assessing sacroiliitis in ankylosing spondylitis: a systematic literature research. Ann Rheum Dis. 2008;67(11): 1535-1540.

9. Jans L, Coeman L, Van Praet L, et al. How sensitive and specific are MRI features of sacroiliitis for diagnosis of spondyloarthritis in patients with inflammatory back pain? JBR-BTR. 2014;97(4):202-205.

10. Pipikos T, Kassimos D, Angelidis G, Koutsikos J. Bone single photon emission/computed tomography in the detection of sacroiliitis in seronegative spondyloarthritis: a comparison with magnetic resonance imaging. Mol Imaging Radionucl Ther. 2017;26(3):101-109.

11. Battafarano DF, West SG, Rak KM, Fortenbery EJ, Chantelois AE. Comparison of bone scan, computed tomography, and magnetic resonance imaging in the diagnosis of active sacroiliitis. Semin Arthritis Rheum. 1993;23:161-176.

12. Geijer M, Gothlin GG, Gothlin JH. Observer variation in computed tomography of the sacroiliac joints: a retrospective analysis of 1,383 cases. Acta Radiol. 2007;48(6):665-671.

13. Biswas D, Bible JE, Bohan M, Simpson AK, Whang PG, Grauer JN. Radiation exposure from musculoskeletal computerized tomographic scans. J Bone Joint Surg Am. 2009;91(8):1882-1889.
14. Klang E, Lidar M, Lidar Z, Aharoni D, Eshed I. Prevalence and awareness of sacroiliac joint alterations on lumbar spine CT in low back pain patients younger than 40 years. Acta Radiol. 2017;58(4):449-455.

15. Geijer M, Gadeholt Göthlin G, Göthlin JH. The validity of the New York radiological grading criteria in diagnosing sacroiliitis by computed tomography. Acta Radiol. 2009;50(6):664-673.

16. Diekhoff T, Hermann KG, Greese J, et al. Comparison of MRI with radiography for detecting structural lesions of the sacroiliac joint using CT as standard of reference: results from the SIMACT study. Ann Rheum Dis. 2017;76(9):1502-1508.

17. Diekhoff T, Hermann KG, Pumberger M, Hamm B, Putzier M, Fuchs M. Dual-energy CT virtual non-calcium technique for detection of bone marrow edema in patients with vertebral fractures: a prospective feasibility study on a single- source volume CT scanner. Eur JRadiol. 2017;87:59-65.

18. Tada K, Ogasawara M, Inoue H, Yamaji K, Kobayashi S, Tamura N. Clinical images: nonradiographic axial spondyloarthritis with sacroiliitis detected by tomosynthesis. Arthritis Rheumatol. 2017;69(8):1706.

19. de Winter J, de Hooge M, van de Sande M, et al. An ASAS-positive MRI of the sacroiliac joints can also occur in healthy individuals, runners and women with postpartum back pain. Arthritis Rheumatol. Epub 2018 Mar 7.

20. Weber U, Lambert RG, Østergaard M, Hodler J, Pedersen SJ, Maksymowych WP. The diagnostic utility of magnetic resonance imaging in spondylarthritis: an international multicenter evaluation of one hundred eighty-seven subjects. Arthritis Rheum. 2010;62(10):3048-3058.

21. Lambert RG, Bakker PA, van der Heijde D, et al. Defining active sacroiliitis on MRI for classification of axial spondyloarthritis: update by the ASAS MRI working group. Ann Rheum Dis. 2016;75(11):1958-1963.

22. Marzo-Ortega H, McGonagle D, O'Connor P, et al. Baseline and 1-year magnetic resonance imaging of the sacroiliac joint and lumbar spine in very early inflammatory back pain. Relationship between symptoms, HLA-B27 and disease extent and persistence. Ann Rheum Dis. 2009;68(11):1721-1727.

23. Althoff CE, Sieper J, Song IH, Haibel H, et al. Active inflammation and structural change in early active axial spondyloarthritis as detected by whole-body MRI. Ann Rheum Dis. 2013;72(6):967-973.

24. Rudwaleit M, van der Heijde D, Landewé R, et al. The development of assessment of SpondyloArthritis International Society classification criteria for axial spondyloarthritis (part II): validation and final selection. Ann Rheum Dis. 2009;68(6):777-783.

25. Rudwaleit M, van der Heijde D, Khan MA, Braun J, Sieper J. How to diagnose axial spondyloarthritis early. Ann Rheum Dis. 2004;63(5):535-543.

26. Slobodin G, Rosner I. Indemonstrable axial spondyloarthritis: does it exist? Isr Med Assoc J. 2013;15(12):780-781.
Journal of Inflammation Research

\section{Publish your work in this journal}

The Journal of Inflammation Research is an international, peer-reviewed open access journal that welcomes laboratory and clinical findings on the molecular basis, cell biology and pharmacology of inflammation including original research, reviews, symposium reports, hypothesis formation and commentaries on: acute/chronic inflammation; mediators of

\section{Dovepress}

inflammation; cellular processes; molecular mechanisms; pharmacology and novel anti-inflammatory drugs; clinical conditions involving inflammation. The manuscript management system is completely online and includes a very quick and fair peer-review system. Visit http://www.dove press.com/testimonials.php to read real quotes from published authors. 